\title{
IMPROVING ACCESS TO CARE FOR PEOPLE WITH SCHIZOPHRENIA AND/OR DEPRESSION IN ARMENIA
}

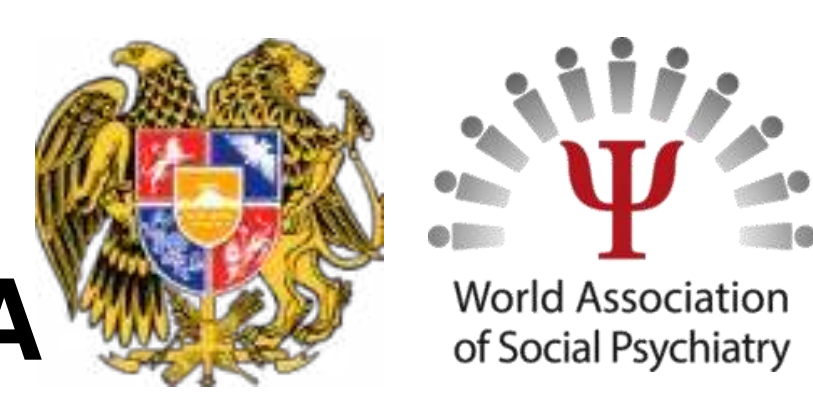

\section{Dr. Elen Aghekyan, Dr. Dicranouhie Ekmekdjan - Yerevan - Armenia}

\section{Objectives}

The objective of this 3-year partnership involving the Ministry of Healthcare of Armenia, the World Association of Social Psychiatry and Sanofi Global Health, is to improve access to care for people with schizophrenia and/or depression in Armenia.

\section{Background and aims}

In Armenia, Mental health care is centralized, hospital-based and with limited psychosocial support ${ }^{1}$. Many mental health facilities, especially in rural areas, lack human resources ${ }^{2}$. Discrimination against people with mental disorder is common in this small population ${ }^{3}$ with $63 \%$ of the total population believing that people with mental disorder are usually violent and dangerous.

Moreover, patients with chronic diseases are nearly three times as likely to get depressed as healthy controls ${ }^{4}$. These are some of the issues that this program aims to address.

\section{Materials and methods}

The specific objectives of the activities initiated in 2017 are:

- Train 600 GPs and 200 nurses on diagnosis and management of schizophrenia \& major depressive disorder (MDD).

- Train 180 non-mental health specialists (oncologists, cardiologists, diabetologists, gynecologists/obstetricians) on MDD.

- Provide CME on MDD and related topics to 120 mental health professionals.

- Raise awareness among the general population to tackle stigma and exclusion.

\section{Outputs and Outcomes (Interim Results - July 2019)}

Training materials (5 modules for schizophrenia \& depression) were developed with WASP and local psychiatrists. WHO mhGAP case studies videos were translated. Training is accredited by National Institute of Health $(\mathrm{MoH})$.

Schizophrenia - 2018: 111 GPs \& 169 nurses trained - Increase in knowledge : $\mathbf{+ 2 1 \%}$ to $\mathbf{+ 8 5 \%}$ (pre \& post training questionnaire).

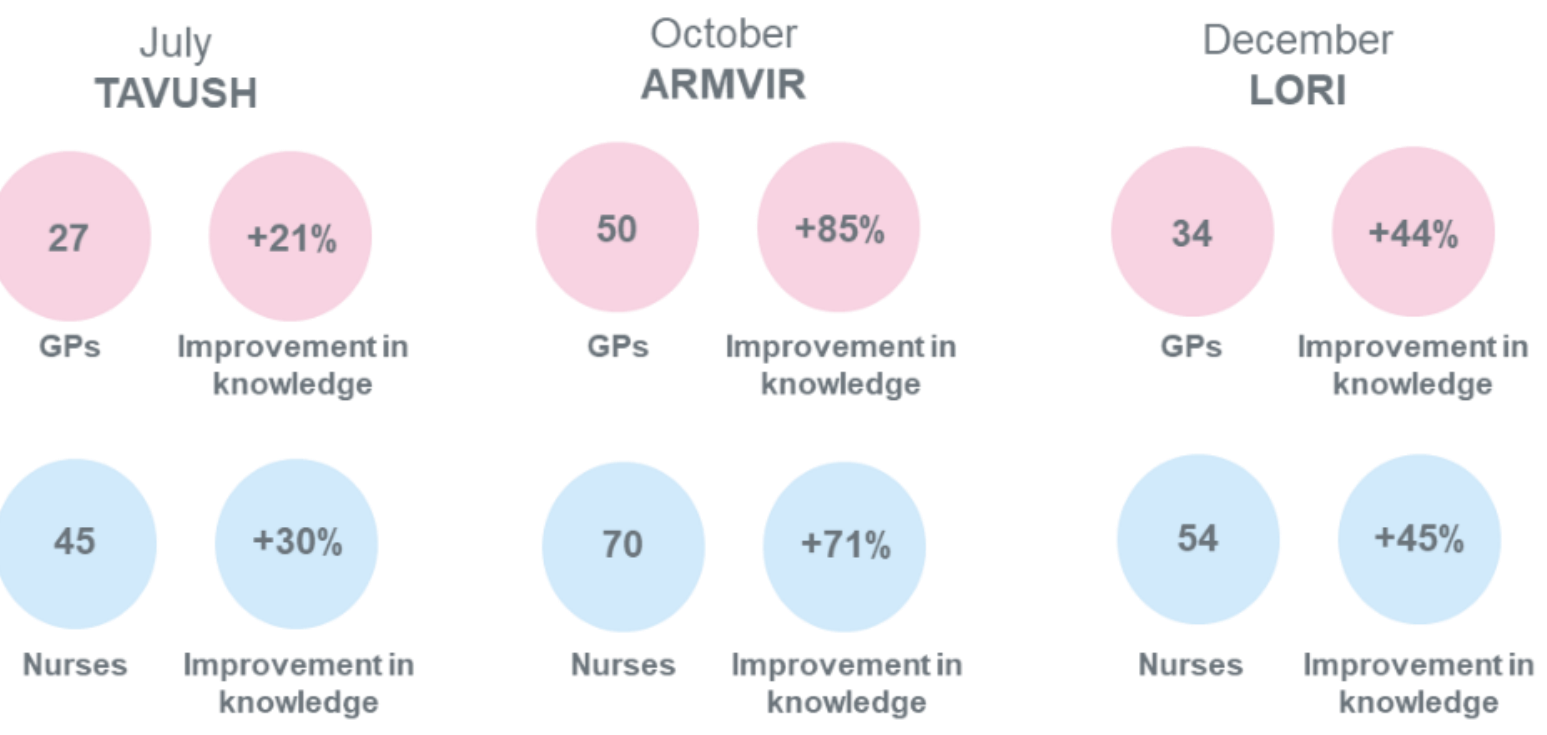

Depression - 2019: 159 GPs trained - Improvement in knowledge

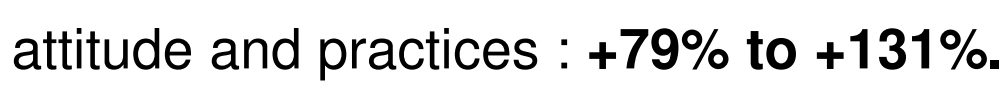

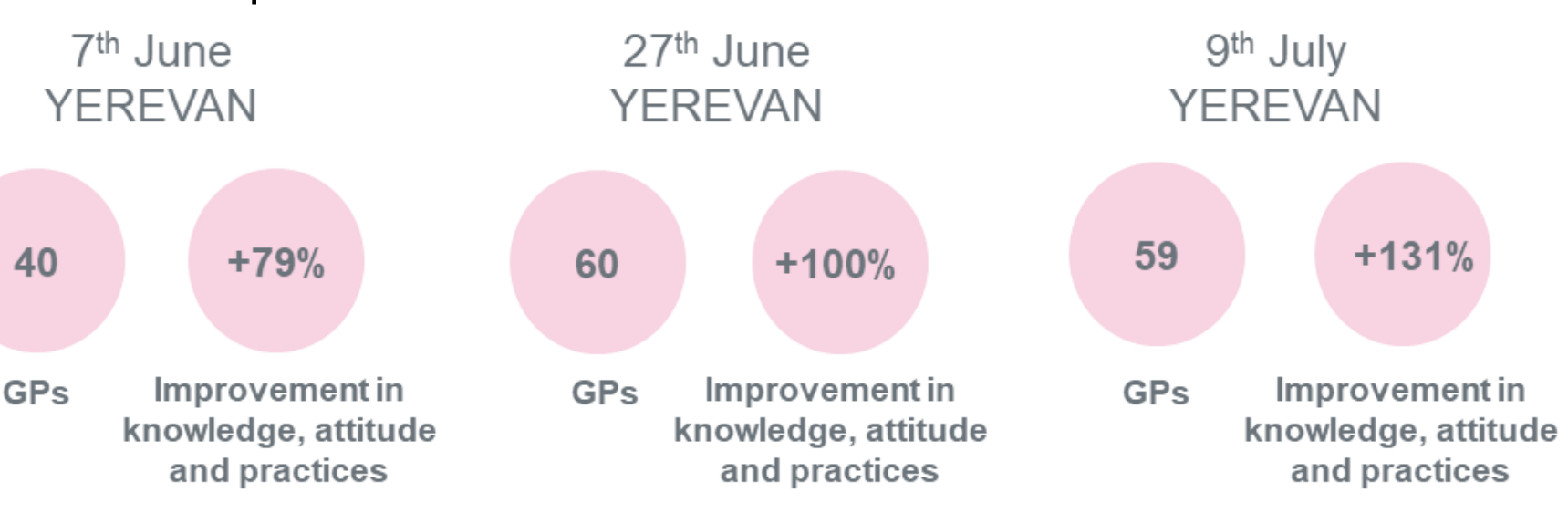

Disease awareness and educational materials on schizophrenia and depression were developed (or adapted): 2 posters, 2 leaflets, 2 comic books, 1 flip-chart.

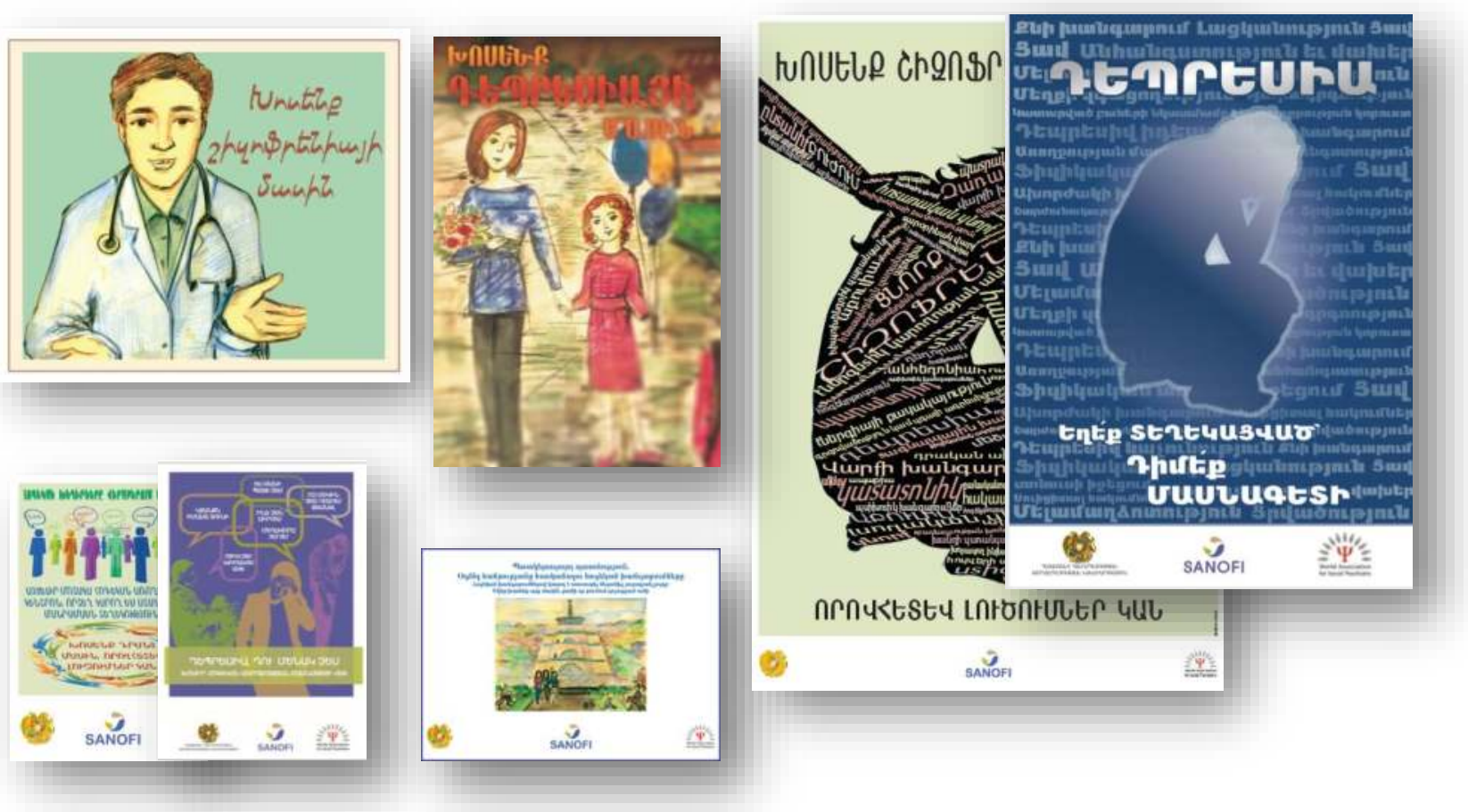

Dissemination of disease awareness and educational materials to $330 \mathrm{HCPs}$ trained on schizophrenia and 159 GPs trained on depression to reach at least est. 310,500 people / year.

Docket system developed and approved by $\mathrm{MoH}$ to assess number of patients identified by trained GPs and referred to psychiatrists is being implemented.
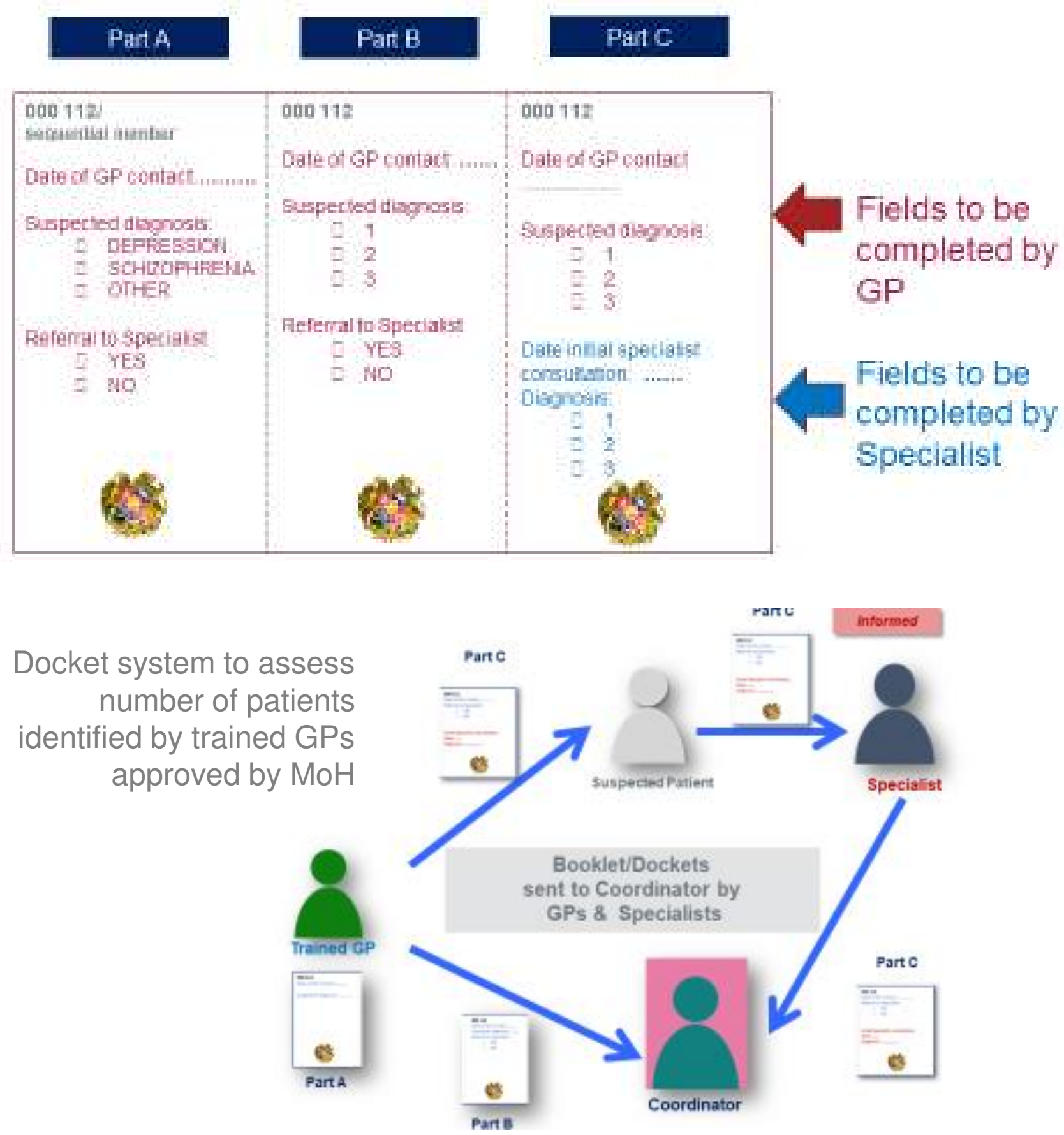

\section{Conclusions}

Despite significant delays due to political changes following the Armenian "Velvet Revolution", the training sessions conducted have already shown positive outcomes in terms of improvement in knowledge, attitudes and practices of GPs and nurses.

However, the main challenge will be evaluating the number of patients identified by trained HCPs and referred to psychiatrists (only possible if docket system is being used). 